Voix et Images

volxetimages

\title{
Fragilités et inquiétude pérenne
}

\section{Robert Major}

Volume 21, numéro 1 (61), automne 1995

Gilles Hénault

URI : https://id.erudit.org/iderudit/201221ar

DOI : https://doi.org/10.7202/201221ar

Aller au sommaire du numéro

Éditeur(s)

Université du Québec à Montréal

ISSN

0318-9201 (imprimé)

1705-933X (numérique)

Découvrir la revue

Citer cet article

Major, R. (1995). Fragilités et inquiétude pérenne. Voix et Images, 21(1),

158-165. https://doi.org/10.7202/201221ar d'utilisation que vous pouvez consulter en ligne.

https://apropos.erudit.org/fr/usagers/politique-dutilisation/ 


\title{
Essai
}

\section{Fragilités et inquiétude pérenne}

\author{
Robert Major, Université d'Ottawa
}

La littérature est toujours un travail sur le fragile.

François Paré

La belle phrase de François Paré, mise en exergue, servira de fil conducteur à cette chronique, qui essayera de rendre compte, non seulement du livre de ce dernier, mais aussi de publications récentes sur Saint-Denys Garneau et Jacques Ferron. Littérature: tous ces mots lancés à la face du silence, depuis la nuit des temps, et qui tentent d'exorciser l'angoisse humaine, exprimant ainsi tant bien que mal l'inquiétude permanente, sans résolution possible, qui est notre condition. Certaines périodes, certains écrivains en témoignent plus éloquemment: témoin la littérature francoontarienne actuelle, voix d'une collectivité qu'on souhaiterait invisible et muette et qui prend la parole dans les marais du silence; témoin également les œuvres de Saint-Denys Garneau et de Jacques Ferron, l'un et l'autre pratiquant une "poétique du désastre "sur fond d'inquiétude pérenne, qui les a conduits au silence et à la mort.

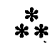

Théories de la fragilité ${ }^{-1}$ se situe résolument dans le prolongement du premier livre de François Paré, Les Littératures de l'exiguité, qui avait remporté un beau succès, bien mérité ${ }^{2}$. François Paré y avait amorcé une réflexion profondément originale sur la littérature et ses marges, le centre et sa périphérie, réflexion allimentée par ses nombreuses lectures d'œuvres appartenant à des littératures marginales et minoritaires, excentriques au sens premier du mot. Cette réflexion, toutefois, était également fondée sur une connaissance approfondie d'une des grandes littératures centripètes, la française (l'auteur étant spécialiste de la Renaissance), et sur des assises théoriques solides, tributaires de l'analyse institutionnelle, qui permettaient à l'auteur d'aborder sans craintes son objet et de tenir à une saine distance critique "la" littérature et son enseignement universitaire. À la fois essai et journal intime, étude savante et plaidoyer, analyse 
critique et manifeste, ce livre s'intéressait à l'espace des restes: ces centaines de littératures minoritaires, coloniales, insulaires, fragiles, qui logent dans les bandes exiguës de la culture occidentale, refoulées, exclues par les grandes littératures hégémoniques.

Théories de la fragilité prolonge cette réflexion, mais en cernant un corpus restreint. Alors que le premier livre embrassait toutes les littératures exiguës et pouvait aussi bien citer des auteurs silésiens ou jamaïcains, basques ou islandais, zaïrois ou occitans, celui-ci s'en tient, à quelques exceptions près, à la littérature franco-ontarienne. "Il m'est devenu impossible de voir la Littérature - toute la littérature, voilà la question - autrement que par les yeux inquiets de ces bandes exiguës de culture, ces écritures de l'exiguïté, qui me semblent bien souvent constituer le tranchant de l'écriture mondialen, avouait l'auteur dans son premier livre ${ }^{3}$. Fidèle à sa démarche, conséquent avec son propos, il nous présente, sous la forme d'une série d'études sur des auteurs franco-ontariens, l'une de ces écritures: celle de sa communauté d'adoption.

Belle occasion pour plusieurs d'élargir leurs lectures ou de simplement prendre connaissance d'une littérature dont les racines remontent au $\mathrm{XIX}^{\mathrm{e}}$ siècle et qui, depuis vingt-cinq ans, se constitue dans son originalité, son autonomie et sa cohérence. Le lecteur québécois, à l'instar de François Paré, lui-même Québécois d'origine mais Franco-Ontarien d'adoption ou d'élection, risque d'être saisi par un foisonnement culturel dont il ne soupçonnait pas l'ampleur, ni l'intérêt. De plus, il y verra de nombreux points de rapprochement avec sa propre littérature: d'une part, parce qu'elles sont contiguës, proches parentes, la littérature franco-ontarienne étant même en partie tributaire de la québécoise, qu'elle accuserait volontiers, d'ailleurs, d'hégémonie; d'autre part, parce que la littérature franco-ontarienne pose, avec une douloureuse acuité, la question de l'identité individuelle et collective, questionnement dont les grandes œuvres québécoises (et en particulier celles dont il sera question dans cette chronique) portent la marque indélébile.

Collection d'études, ce volume n'est pas un livre de théorie littéraire et le titre pourrait en abuser plusieurs. Il faut prendre "théorie" dans un de ses sens, rare, dit "littéraire" selon Le Robert: "groupe de personnes qui s'avancent les unes derrière les autres. Voir: cortège, défilé, procession." Voici donc un défilé d'auteurs, mais dont la procession solennelle est accompagnée néanmoins d'une réflexion théorique sur les conditions d'écriture dans un contexte de faiblesse, d'exclusion, de domination, quand le sujet angoissé se sent membre d'un groupe dont la fragilité est presque morbide.

Les auteurs étudiés par François Paré ont pour noms Fernand Dorais, André Paiement, Paul Savoie, Jean Marc Dalpé, Pier Rodier et Marie-Thé Morin, Michel Ouellette, Daniel Poliquin, Michel Marc Bouchard, Andrée Christensen, Gérard Bessette. Se greffent à ce corpus central un nom québé- 
cois, Saint-Denys Garneau, et deux Acadiens, France Daigle et Herménégilde Chiasson, ce dernier étant le seul "étranger" à être étudié longuement.

Cette liste pose crûment la question de l'identité franco-ontarienne. Question complexe, les lieux d'appartenance des auteurs pouvant être multiples, et difficilement résoluble, chacun constituant un cas d'espèce. Certains auteurs - pour ce qui est de leur fiche signalétique, du moins sont aisément classables. Ainsi un Daniel Poliquin, écrivain de souche, né à Ottawa, dessinant, d'une œuvre à l'autre, une géographie symbolique de l'Ontario français, approfondissant ce que cela signifie d'être minoritaire, incertain de son identité, réduit à un discours inquiet. Mais de telles fiches d'identité sont relativement rares dans cette littérature. Les uns, comme Fernand Dorais, sont des Montréalais exilés, encore que ce dernier se soit passionnément identifié à la collectivité franco-ontarienne au point d'élaborer, dans ses essais, ce que François Paré appelle une "anthropologie de la minorisation"; 'd'autres, comme Paul Savoie, ont pu rester profondément marqués par une enfance vécue ailleurs, manitobaine dans son cas. De plus, ce dernier publie autant en anglais qu'en français (ce que ne mentionne pas François Paré!) et édite au Manitoba et au Québec (pour ce qui est des œuvres françaises, du moins). Dans quelle mesure est-il un auteur franco-ontarien? Et que dire de Gérard Bessette, que l'institution littéraire québécoise hésiterait à sacrifier, même s'il a passé une bonne partie de sa vie en Ontario et même si son œuvre indique par de nombreuses facettes son appartenance à sa communauté d'accueil. Bessette se reconnaît "en quelque sorte" écrivain francoontarien. François Paré, tout en admettant que la recherche des étiquettes institutionnelles reste oiseuse, se fait fort, toutefois, de montrer que l'œuvre bessettienne (par sa diglossie, par son hybridité, par l'étrangeté du sujet minorisé) marque elle-même très clairement son lieu d'insertion.

Avec raison, l'auteur 'ne s'attarde pas à cette question des étiquettes. Il lit et commente des œuvres qui lui semblent exemplaires d'une recherche éperdue d'une identité individuelle et collective. C'est d'ailleurs la forte présence d'une conscience collective ou, exprimée autrement, la conviction que la littérature ne saurait vivre en niant les forces vives de la communauté ${ }^{4}$ qui donne à ces œuvres leur accent inimitable.

Si les littératures minoritaires restent si profondément chargées de sens pour moi, c'est justement qu'elles tendent à poser clairement et douleureusement la question de l'identité collective, et donc celle de la participation de l'écriture à la violence manifestée et aux processus d'exclusion, d'une part, et à la fête, à l'inclusion, à la procession, d'autre part. (p. 13)

Les mots "violence" et "exclusion" ne sont pas excessifs. Le Franco-Ontarien sait, de naissance, qu'il est invisible, qu'on le souhaite tel et que rompre cette invisibilité l'expose.

Ne suffit-il pas d'avoir un jour commandé en français dans un train ou un avion au Canada un vulgaire café ou autre friandise en soi sans importance 
pour sentir dans le visage du préposé anglophone l'intensité de la différence qui s'exaçerbe en nous? Et cette criante différence (elle est pourtant si fragile!) vient toujours briser, à ce qu'il nous semble, le ronronnement, la douceur opiniâtre de l'unanimité autour de nous. L'air, dans ces cas-là, n'est plus tout à fait le même. En nous, l'invisibilité s'est exposée au grand jour. Il y a un embarras vague, une petite hésitation, un chuchotement. Le geste brusque, le sourire crispé, la rancœur à peine dévoilée quand se pose sur la tablette le très simple objet du désir révèlent mieux que toute autre chose l'affirmation initiale, scandaleuse, de l'intolérable mais infime différence que nous avons résolu d'être ce jour-là, que nous sommes à tous les jours, que nous avons toujours été sur ce continent. (p. 23)

C'est dans un tel contexte de fragilité que l'écrivain franco-ontarien prend la parole.

D'où le caractère éminemment oral, excessif, iconoclaste de la première génération d'écrivains et d'animateurs culturels qui, au début des années soixante-dix, à Sudbury, marquèrent avec violence leur refus des conditions d'oppression et de stagnation. André Paiement, Pierre Bélanger, Robert Dickson, Gaston Tremblay, Brigitte Haentjens, Marcel Aymar, Fernand Dorais sont les acteurs importants de cette naissance de la littérature franco-ontarienne. La chanson, le théâtre, la poésie connaissent une explosion remarquable, bien appuyés par les instruments de leur diffusion: maison d'édition (Prise de parole), théâtre (Théâtre du Nouvel-Ontario), coopérative ou commune de créateurs (CANO: Coopérative des artistes du Nouvel-Ontario). Sudbury, ville minière, ville prolétarienne, ville nordique et excentrique, mais aussi ville universitaire, devient le berceau et le haut lieu de la culture franco-ontarienne. Le théâtre d'André Paiement, fragmenté, décousu, violent, essentiellement oral et réfractaire à l'écriture, communautaire tout autant dans son inspiration et sa genèse que dans sa visée, est tout à fait représentatif de cette période. Paiement lui-même, d'ailleurs, personnage public et animateur charismatique, exercera une énorme influence, au-delà de sa mort prématurée en 1978, et d'autant mieux que celle-ci "a été immédiatement prise en charge et sacralisée par plusieurs de ses contemporains et amis" (p. 66). Fernand Dorais, quant à lui, sera le théoricien et l'analyste de cette période, la décrivant avec une sensibilité d'écorché vif tout en étant porté, malgré tout, par une foi quasi théologale dans les vertus rédemptrices de cette fête communautaire.

François Paré insiste fort judicieusement sur cette naissance vociférante et apparemment spontanée et les chapitres qu'il consacre à André Paiement et à Fernand Dorais sont parmi les plus réussis du livre. Mais il montre aussi les limites de cette période de refus. Les notions de norditude, d'oralité, de classe, d'aliénation dessinent rapidement leurs limites, en particulier l'oralité, par son rejet d'une langue (trop) écrite, qui met en question l'existence même d'une littérature.

Ce refus ambigu a encore cours au théâtre (Jean Marc Dalpé, Michel Ouellette), mais dans les années quatre-vingt-dix, c'est Ottawa qui devient 
le centre de la production littéraire et une seconde génération d'écrivains, romanciers et poètes, donnent une tout autre impulsion à la littérature franco-ontarienne. Les romans de Daniel Poliquin, les poèmes de Paul Savoie et d'Andrée Christensen, entre autres, montrent l'extrême diversité et la grande richesse de la production actuelle. L'œuvre de Daniel Poliquin, à cause du succès de L'Écureuil noir, est mieux connue des lecteurs québécois. Celle d'Andrée Christensen (quatre livres depuis 1990) et de Paul Savoie, sans doute moins. L'analyse qu'effectue François Paré de chacune de ces œuvres leur rend justice et laisse entrevoir la complexité d'univers imaginaires qui méritent de nombreux lecteurs.

François Paré, d'ailleurs, est très conscient de faire quasiment œuvre de pionnier, avec les limites intrinsèques de cette démarche.

J'ai déjà accusé ailleurs l'institution littéraire de trahir l'importance d'œuvres comme celles de Daniel Poliquin, d'André Paiement, de France Daigle ou d'Herménégilde Chiasson. Ces œuvres n'avaient pas bénéficié de l'attention critique qu'elles méritaient; elles n'avaient jamais été placées dans une entreprise de connaissance que je voyais comme la fonction primordiale de la littérature. Ainsi, il a bien fallu commencer [...] (p. 139)

L'entreprise de connaissance est donc lancée.

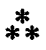

L'œuvre de Saint-Denys Garneau, quant à elle, est déjà bien connue. Depuis sa mort prématurée et mystérieuse, le poète a été l'objet d'une attention constante: on est passé de la quasi-hagiographie de ses contemporains au refus sardonique des tenants de la contre-culture, pour finalement en venir à la patiente investigation de la critique universitaire. Le cinquantième anniversaire de la mort de Garneau a occasionné, en 1993, toute une série de manifestations. Voix et Images y a fait écho en publiant, comme dossier du numéro 58 , les actes d'un colloque tenu à l'Université d'Ottawa. Un autre colloque, tenu à l'Université de Montréal, est la matière du volume Saint-Denys Garneau et La Relèves.

L'originalité de ce collectif réside en la volonté des collaborateurs d'examiner les rapports de Saint-Denys Garneau avec ce lieu d'un discours particulier que fut $\mathrm{La}$ Relève. D'emblée le problème est posé : «L'une des questions les plus difficiles s'offrant à l'histoire culturelle est celle des rapports entre la production des écrivains et celle des groupes auxquels ils ont appartenu ou ont été liés" (p. 7). Question fascinante, en effet. Pour l'avoir posée, jadis, au sujet des écrivains de Parti pris, je sais qu'il n'est guère aisé de lier la production effective d'un écrivain d'envergure au programme ou à l'idéologie d'un groupe. Entre les relations de droit et de fait s'insèrent toute une série de médiations complexes, sources d'ambiguités et de contradictions. C'est le lieu même de l'écriture, qui 
questionne toutes les visions du monde, y compris celle dont elle se réclame. Les collaborateurs à ce volume, héritiers d'une déjà longue tradition critique garnélienne, s'en tiennent donc pour l'essentiel à cette problématique, tout en faisant leur profit des nombreuses études existantes sur la conscience inquiète du poète et sur son œuvre à la fois si étrange et si contemporaine.

Ainsi, Michel Biron, après avoir noté que Saint-Denys Garneau "semble avoir appartenu à la seule Relève" (p. 11), et qu'on ne peut l'imaginer en dehors de ce lieu discursif, s'attache à dégager ce qui démarque le poète de cette appartenance. Sociologiquement, Saint-Denys Garneau appartient au groupe par des liens ténus et ambigus. Mais c'est sa poésie, surtout, qui marque des dissonances. Dans sa forme textuelle et dans son vocabulaire, celle-ci se passe de la référence catholique. Surtout, elle s'expose aux fissures et les recherche, ne souhaite aucunement restaurer un ordre antérieur et une plénitude d'être, mais au contraire veut habiter le monde moderne, sans nostalgie, tel qu'il est.

Par ailleurs, Pierre Nepveu scrute l'horizon américain de Saint-Denys Garneau et s'étonne de constater que l'Amérique est totalement absente du vocabulaire du poète alors même qu'elle devient "une question, un problème " pour les contemporains du poète, l'objet d'un discours' de plus en plus riche et animé. L'Amérique mythique, l'Amérique rêvée fascinent Le Moyne, Laurendeau et Charbonneau, comme de nombreux contemporains de La Relève. Saint-Denys Garneau n'en fait aucune mention. Silence réel ou présence de cet envers de l'Amérique, de cette autre Amérique, celle d'Emily Dickinson, celle de la "voix privée, petite, tranquille du poète ?

André Brochu, pour sa part, effectue une analyse de la problématique de l'intériorité dans l'œuvre de Saint-Denys Garneau au moyen d'une série de comparaisons révélatrices avec ses contemporains. L'opposant d'abord à la perception d'Ernest Gagnon qui, dans L'Homme d'ici, valorise "l'homme d'ici" (l'homme de l'intériorité, de la contemplation) au détriment de "l'homme de là (l'homme d'action, l'homme de raison); cette dualité sert ensuite à marquer les ressemblances et dissemblances entre Saint-Denys Garneau, d'une part, et Robert Charbonneau, Robert Élie et Jean Le Moyne, d'autre part. À partir de ces comparaisons se dégage l'originalité du poète face à ses amis et compagnons : à la fois partiellement réfractaire à cette dualité, plus complet, conscient de ses insuffisances et sachant s'en nourrir.

C'est aussi à une comparaison que se livre Élisabeth Nardout-Lafarge: cette fois entre le poète et Berthelot Brunet, tous deux lecteurs des auteurs français, en insistant, toutefois, sur la lecture du second. D'autres lectures sont présentées dans les études suivantes: les lectures québécoises du philosophe Jacques Maritain, alors qu'Yvan Cloutier dresse un 
tableau de son influence au Québec et en particulier auprès de La Relève; les lectures cléricales (fort réduites, au demeurant) du recueil Regards et Jeux dans l'espace, ce qui permet à Jane Everett de dégager les limites de cette réception critique; la lecture qu'effectue Saint-Denys Garneau de sa propre œuvre, qui porte Paul Chamberland à postuler que ce lecteur interne dérobé, au regard oblique, est en réalité un surmoi accusateur et persécuteur qui fera échec au processus créateur et condamnera le poète au silence. Finalement, Lucie Bourassa amorce une réflexion sur le temps discursif en confrontant quelques énoncés thématisant le temps dans la poétique et dans la poésie de Garneau avec la temporalité discursive d'un poème en particulier. C'est donc sur une analyse technique du texte luimême que se clôt ce recueil d'une belle tenue.

Le collectif sur Jacques Ferron ne contient pas les actes d'un colloque mais plutôt une série d'études et de documents émanant d'un groupe de recherche dirigé par Ginette Michaud. L'Autre Ferron ${ }^{6}$ est donc beaucoup plus volumineux et présente côte à côte des collaborations de critiques chevronnés et d'étudiants. Les admirateurs de Jacques Ferron seront aussi heureux d'y trouver toute une série d'inédits, bien commentés: des fragments du Pas de Gamelin, des extraits de correspondances avec Clément Marchand et avec le traducteur Ray Ellenwood, ainsi que des transcriptions d'entretiens de l'automne 1982 avec Pierre L'Hérault. Un choix bibliographique "depuis 1980" complète l'ensemble.

Sans doute le titre du volume en étonnera-t-il quelques-uns. Pour qu'il y ait un "autre" Ferron, encore faudrait-il qu'il y en ait eu d'abord "un", qui vraisemblablement aurait fait l'unanimité jusqu'à présent. Or, l'œuvre diverse, incroyablement tortueuse et complexe de Jacques Ferron semble se jouer de toutes ces tentatives de résolution; constituée d'un réseau inextricable de souvenirs et de nostalgies, coulés au creuset d'abondantes lectures et d'un imaginaire mythologique, elle se présente le plus souvent sous forme de rébus ou de paradoxe, que se plait, d'ailleurs, à obscurcir l'auteur lui-même par les confidences et les commentaires qu'insidieusement il prodigue si généreusement. Plus qu'aucun autre écrivain québécois - et peut-être n'est-ce qu'un effet de son style allusif, elliptique et ironique - Ferron a construit son mystère. Les nombreux ferroniens en ont donc sur la planche pour plusieurs années encore.

Mais cela, les collaborateurs à ce volume le savent mieux que quiconque. Aussi, plutôt que de leur reprocher ce choix de titre, reconnaissons qu'ils présentent un Ferron protéiforme et multiple, qui s'ajoute aux autres que nous connaissions déjà. Marcel Olscamp, excellent biographe, étudie les années gaspésiennes, antérieures à la vocation publique mais si importantes pour la formation de l'écrivain; Jean-Pierre Boucher effectue une distinction précise entre les deux recueils de contes maintenant toujours édités conjointement; François Chaput dégage les éléments épiques 
dans Le Ciel de Québec; Pierre L'Hérault se livre à une analyse méticuleuse du Saint-Élias; Pascale Sirard suit à la trace le mythe de Narcisse dans l'œuvre de Ferron; Ginette Michaud est retenue par le rayon anglais de la bibliothèque imaginaire de l'auteur alors que Betty Bednarski revient sur les figures de l'anglicité dans l'œuvre; finalement, Patrick Poirier examine l'échec que fut $L e$ Pas de Gamelin. Ces études sont bien menées, étoffées (quelquefois touffues). L'ensemble, inédits et études, forme un volume irremplaçable.

La question de l'identité, identité chercheuse et inquiète, parvenant difficilement à se dire, est au centre de la lecture que fait François Paré de la littérature franco-ontarienne. Lui-même établit le lien avec les poèmes de Saint-Denys Garneau : "Et c'est à Saint-Denys Garneau que j’ai songé soudainement, lui que l'impuissance avait semblé habiter si douleureusement. N'est-il pas à vrai dire, dans l'univers de la parole difficile - celle dont il est souvent question dans ce livre - , mon cousin le plus ancien, le plus obstiné?" (p. 38)

Il aurait pu tout aussi bien établir la relation avec l'œuvre de Jacques Ferron, tout entière axée sur l'existence du pays incertain et exprimant ses inquiétudes sur. la perennité de ce pays et la survivance de la petite bibliothèque où se glisseraient ses livres ${ }^{7}$.

Tous travaillant sur le fragile.

1. François Paré, Théories de la fragilité, Ottawa, Le Nordir, 1994, 156 p.

2. Prix littéraire du Gouverneur général 1993 et Signet d'or 1993 de Radio-Québec. Sur ce livre, voir la *Chronique * de l'hiver 1993 (Voix et Images, $\mathrm{n}^{\circ}$ 54).

3. François Paré, Les Littératures de l'exiguité, Hearst, Le Nordir, 1992, p. 7.

4. François Paré utilise, quant à lui, les mots "communal " et "communalité" (sic) tout au long de son essai, sans expliquer ni justifier cet usage.

5. Benôt Melançon et Pierre Popovic (dir.), Saint-Denys Garneau et La Relève, Montréal, Fides, coll. a Nouvelles études québécoises ", 1995, 133 p.

6. Ginette Michaud (dir.), L'Autre Ferron, avec la collaboration de Patrick Poirier, Montréal, Fides, coll. "Nouvelles études québécoises ", 1995, 468 p.

7. Voir l'entretien avec Pierre L'Hérault, L'Autre Ferron, op. cit., p. 406-407. 